\title{
Time-dependent formation of the profile of resonance atomic states and its dependence on the duration of ultrashort pulses from free-electron lasers
}

\author{
C. A. Nicolaides, ${ }^{*}$ Th. Mercouris, ${ }^{\dagger}$ and Y. Komninos ${ }^{\ddagger}$ \\ Theoretical and Physical Chemistry Institute, National Hellenic Research Foundation, \\ 48 Vasileos Constantinou Avenue, Athens, 11635 Greece \\ (Received 22 July 2009; published 12 November 2009)
}

\begin{abstract}
We have computed from first principles the dependence of the formation of the resonance profile of the $\mathrm{He} 2 s 2{ }^{1} P^{o}$ state at $60.1 \mathrm{eV}$ on the duration of the interaction of $\mathrm{He} 1 s^{2}{ }^{1} S$ with ultrashort Gaussian electromagnetic pulses for field strengths smaller than 0.04 a.u. The calculations took into account the self-consistent field, the state-specific electron correlations and the mixing of bound with scattering components. Apart from the new physical information that they reveal, such results, and similar ones for other resonance states, can be of use to current developments toward the accurate characterization of ultrashort pulses from free-electron lasers in the regimes of a few femtoseconds to a few hundreds of attoseconds.
\end{abstract}

DOI: $10.1103 /$ PhysRevA.80.055402

PACS number(s): 32.80.Rm, 31.15.A-

A standard desideratum in the science and technology of light production is the accurate knowledge of the characteristics of the radiation pulses. In recent years, this aim has been associated with the production of ultrashort pulses, the duration going down to the range of a few femtoseconds (fs) and of a few hundreds of attoseconds (as). In this context, of special current interest is the possibility of having accurate diagnostics for the ultrashort pulses from free-electron lasers (FELs), e.g., [1-9]. Indeed, although the characterization of such pulses in terms of energy or intensity is achievable with accuracy, the reliable and consistent determination of their exact shape and duration remains a problem that exhibits practical experimental difficulties.

The purpose of this Brief Report is twofold:

First, to report on the theory and computation of accurate numbers for the time-dependent formation via photoabsorption of a well-known resonance state, the He $2 s 2 p{ }^{1} P^{o}$, as a function of the ultrashort duration of pulses with Gaussian temporal shape.

Second, to suggest that such information from theoretical atomic physics may serve as reference data for the calibration of results and techniques of production of ultrashort FEL pulses.

The photon energies in such pulses range, from, say, a few tens to many hundreds (for our purposes) of eV. This means that their interaction with atoms and molecules always creates ionized species even by one-photon absorption. Inside such continua there are resonance states corresponding to multiply or inner-hole excited configurations. The electron correlations in such configurations lead to mixing of bound and scattering components, with the result that the photoexcitation cross-section contains distinct features over the smooth background of the continuous spectrum, (e.g., asymmetric peaks), which are due to these resonance states. It is aspects of these features and of their variation with respect to pulse characteristics that we have analyzed and computed, and that we report here.

\footnotetext{
*caan@eie.gr

†thmerc@eie.gr

†ykomn@eie.gr
}

In previous work $[10,11]$, we demonstrated how it is possible to compute from first principles, for weak-as well as for strong-field pulses, the time-dependent formation of the profile of valence multiply excited or of inner-hole resonance states.

The theory is structured so as to be able to handle arbitrary electronic structures, accounting for the state-specific self-consistent fields and electron correlations in the discrete and in the continuous spectrum.

Here we opine that if the information which is obtained from such investigations is numerically accurate, then it can be used directly in current research on FELs, not only as a possible common domain of theoretical and experimental applications but also for the purpose of facilitating the numerically accurate determination of experimental pulse parameters such as intensity, shape, and duration.

The atomic physics part of the proposal is demonstrated here by considering from first principles the time-dependent dynamics of the photoabsorption process

$$
\mathrm{He} \Psi_{i}\left(1 s^{2}{ }^{1} S\right) \underset{\text { one-photon absorption }}{\stackrel{\text { FEL ultrashort pulse }}{\longrightarrow}} \mathrm{He} \Psi_{E}(2 s 2 p, 1 s E p)
$$

for energies in the vicinity of $60.1 \mathrm{eV}$ where the doublyexcited He $2 s 2 p{ }^{1} P^{o}$ autoionizing state exists $[10,12,13]$ dissolved into the $1 s E p$ continuum of scattering states. The intensity of the pulse is assumed to be weak (see below). In this work, the wave functions for the $\operatorname{He} \Psi_{i}\left(1 s^{2}{ }^{1} S\right)$ and for the $\mathrm{He}_{E}(2 s 2 p, 1 s E p)$ states which enter into the expansion for the $a b$ initio solution of the time-dependent Schrödinger equation (TDSE) have been computed via the state-specific approach $[10,11]$, and references there in.

When, instead of an ultrashort pulse, process (1) occurs due to broad-band excitation by radiation of weak intensity, the problem of analyzing the formation of the resonance involves only the resolution on the energy axis, either experimentally or theoretically. Indeed, this problem has constituted the standard example of interference in the photoionization cross section, whose resonance profile was first predicted by Fano in the form $[12,13]$, 


$$
\sigma(E)=\sigma_{a} \frac{(q+\varepsilon)^{2}}{1+\varepsilon^{2}}+\sigma_{\text {background }}(E), \quad \varepsilon=(2 / \Gamma)\left(E-E_{r}\right)
$$

and was verified in the synchrotron radiation experiments of Madden and Codling [13]. In Eq. (2), $\Gamma$ is the linewidth [full width at half maximum (FWHM)], $E_{r}$ is the resonance energy, and $q$ is the asymmetry parameter [12].

When photoabsorption involves the interaction of an atomic (molecular) state with an ultrashort pulse rather than with continuous radiation, Eq. (2) must be replaced appropriately. Furthermore, the overall theoretical and computational framework must be time as well as energy dependent.

The first demonstration of how to handle quantitatively from first principles the computation of the time-dependent probability of the formation of the resonance profile for process (1), (and of similar ones), was published recently [10]. It includes both an analytic methodology which is based on first order time-dependent perturbation theory (FOTDPT) and is valid for weak pulses, and a numerical one which solves nonperturbatively, via the state-specific expansion approach (SSEA), the polyelectronic TDSE and is valid for weak as well as for strong pulses $[10,11]$.

The fundamental quantity which is related to the timedependent profile of resonance formation in process (1), is the differential ionization probability,

$$
P(E, t)=|\langle 1 s E p \mid \Psi(t)\rangle|^{2}
$$

The characteristics of the pulse are encoded in the information produced by $P(E, t),|\Psi(t)\rangle$ being the solution of the TDSE in the presence of the pulse-atom interaction in the dipole approximation.

For the purposes of the present study, $P(E, t)$ was computed as a function of pulse duration using both the analytic results of [10] and the $|\Psi(t)\rangle$ that is obtained from the nonperturbative (numerical) solution of the TDSE. As in every theoretical analysis, the analytic results in general facilitate the extraction of quantitative and transparent information for the domain of their applicability. In the present case, our earlier study [10] has shown that the value of the field strength at which the FOTDPT starts losing accuracy for this problem is $F=0.04$ a.u., (Fig. 2 of [10]). For smaller values, we are in the regime of the weak field. This is the regime for which the present study was carried out. In work which is in progress, we also examine aspects of the time-dependent formation (or distortion and destruction) of resonance states when the intensity of the short pulse is strong, so as to produce reliable data on the resulting distortion of the resonance profile as a function of intensity.

The calculations were carried out for pulses of two shapes: the sine-square, $\sin ^{2} \frac{2 \pi t}{T},(T$ is the duration of the pulse), and the Gaussian. The sine-square pulse was used in [10], where it was shown how, for a fixed (long) pulse duration, the profile is being built as a function of time in the regime of attoseconds and of a few femtoseconds. (Fig. 3 of [10]).

Here, we report only the results that were obtained using Gaussian pulses, (Figs. 1-3), since we expect that these rep- resent the experimental situation more faithfully than the sine-square pulses do.

Our calculations of the $|\Psi(t)\rangle$ which is needed in Eq. (3) were done for the Hamiltonian $H(t)=H^{A}+V(\omega, t)$, where $H^{A}$ is the free atom Hamiltonian and $V(\omega, t)=z F g(t) \sin (\omega t)$. $\omega$ is the frequency, $g(t)$ is the temporal shape of the pulse while the field is linearly polarized along the $z$ axis. The energydependent wave functions for the stationary scattering state were obtained by solving the free-electron Schrödinger equation in the field of the $\mathrm{He}^{+} 1 s$ ion. The results of physical interest are those for which the parameter of time, i.e., of the point in time at which measurement of the intensity of photoelectrons takes place, is much larger that the duration of the pulse.

In the framework of FOTDPT, using the function $G(E, t)$ defined by, ( $E_{i}$ is the energy of the initial state),

$$
G(E, t)=-i F \int_{-\infty}^{t} d t^{\prime} e^{-i\left(E_{i}-E\right) t^{\prime}} g\left(t^{\prime}\right) \sin \omega t^{\prime}
$$

the amplitude for excitation, $A_{E}(t)$, is obtained as [10],

$$
A_{E}(t)=C_{E} e^{-i E t} G(E, t)[q+\varepsilon]-C_{E} \frac{q-i}{2 \pi i} e^{-i z_{0} t} G\left(z_{0}, t\right)
$$

with

$$
P(E, t) \equiv\left|A_{E}(t)\right|^{2}
$$

The symbols $q$ and $\varepsilon$ in Eq. (5) were defined above. In addition,

$$
C_{E}=\frac{(\Gamma / 2) d\left(E_{r}\right)}{\sqrt{\left(E-E_{r}\right)^{2}+(\Gamma / 2)^{2}}},
$$

where $d\left(E_{r}\right)$ is the value of the dipole matrix element at $E_{r}$. The symbol $z_{0} \equiv E_{r}-i \Gamma / 2$ is the complex eigenvalue of the resonance state.

As already said, our analytic and numerical results were obtained for $g(t)=e^{-\left(t-t_{0}\right)^{2} / 2 \tau^{2}}$. The parameter $\tau$ is given by, $\tau=\frac{t_{F W H M}}{2 \sqrt{\ln 2}}$, where the time $t_{F W H M}$ corresponds to the full width of the intensity at half maximum, i.e., it is directly related to experimental conditions. All the results given here involve $t_{F W H M}$.

Using the Gaussian $g(t)$, in the rotating wave approximation, where the energy-nonconserving terms are discarded, the function $G(E, t)$ of Eq. (4) is found to be

$$
G(E, t)=\sqrt{2 \pi} \tau F e^{-1 / 2(\delta E)^{2} \tau^{2}} e^{i \delta E t_{0}} \frac{1}{2}\left[1+\operatorname{erf}\left(\frac{t-t_{0}}{\sqrt{2} \tau}-i \frac{\delta E \tau}{\sqrt{2}}\right)\right]
$$

with $\delta E \equiv E-E_{i}-\omega$.

$G(E, t)$ contains the information on the pulse, which is combined with that of the resonance state in the $A_{E}(t)$ of Eq. (5). Note that, for $t \gg t_{0}+\sqrt{2} \tau$ the value of the error function approaches unity, and so for photon energies very close to the resonance state energy, 


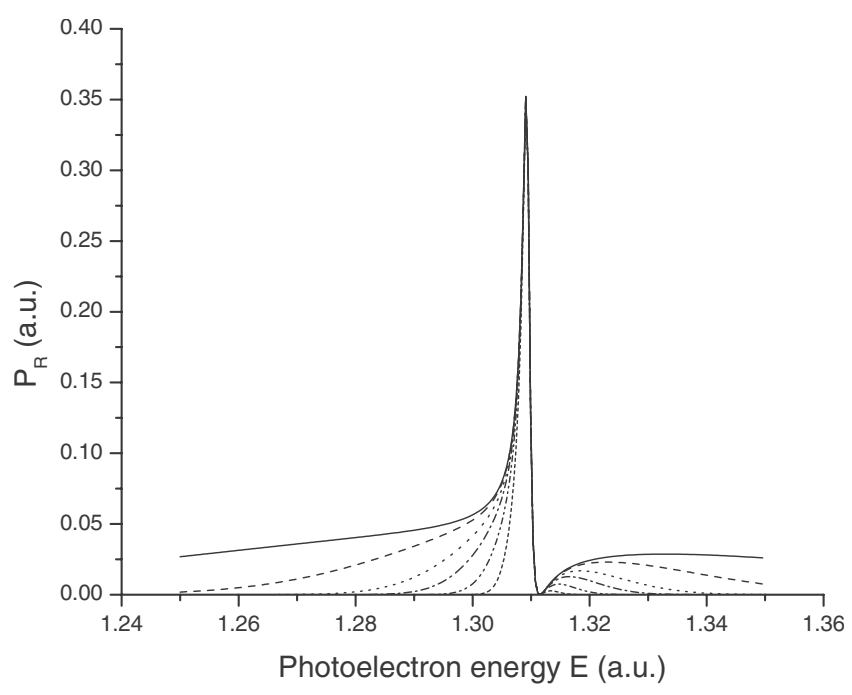

FIG. 1. The "reduced" differential ionization probability, Eq. (10), for different values of $t_{F W H M}$ for weak-field pulses. Solid line: $t_{F W H M}=484$ as. Dashed line: $t_{F W H M}=1.21$ fs. Dotted line: $t_{F W H M}$ $=2.42$ fs. Dash-dot line: $t_{F W H M}=3.63$ fs. Dash-dot-dot: $t_{F W H M}$ $=6.05$ fs. Short dash: $t_{F W H M}=10.89$ fs.

$$
P(E, t) \approx 2 \pi \tau^{2} F^{2} d^{2} e^{-\left(1 / 2 \Gamma \varepsilon+\delta E_{0}\right)^{2} \tau^{2}} \frac{(q+\varepsilon)^{2}}{1+\varepsilon^{2}}
$$

for

$$
t \gg t_{0}+\sqrt{2} \tau
$$

with $\delta E_{0}=E_{r}-E_{i}-\omega$.

Equation (9) exhibits the modification of Eq. (2) which is caused by the time-dependent Gaussian shape of the interaction.

Here, it is appropriate to stress that the energy dependence of $P(E, t)$, in Eq. (9), is not restricted to only one point, $E$ $=E_{i}+\omega$, since irradiation by an ultrashort pulse excites states in a very broad energy spectrum.

Given the possibility of computing accurately $P(E, t)$ for an atomic state of our choice, we wish to identify quantities that can be related to experimental variations of pulse parameters. We have already assumed a Gaussian shape and a weak field, i.e., strengths below 0.04 a.u., for photon energies around $60.1 \mathrm{eV}$. We now turn to Eqs. (5)-(9). From these it follows that $P(E, t)$ is proportional to $F^{2} t_{F W H M}^{2}$. Therefore, we have defined a "reduced" differential ionization probability

$$
P_{R} \equiv \frac{P(E, t)}{F^{2} t_{F W H M}^{2}}, \quad \text { for } t \gg t_{0}+\sqrt{2} \tau
$$

and computed it as a function of $t_{F W H M}$.

The results for $P_{R}$ for a number of values of $t_{F W H M}$, ranging from the attosecond to the few-femtosecond regime, (which is of current experimental interest, e.g., [1-9]), are shown in Fig. 1. This function has the following two relevant characteristics. There is an intense and sharp maximum very near the exact resonance position, since the value of $q$ is relatively small. (The value of $q$ is about $-2.8[10,12,13]$ ). The other characteristic is the broad extension to the right of

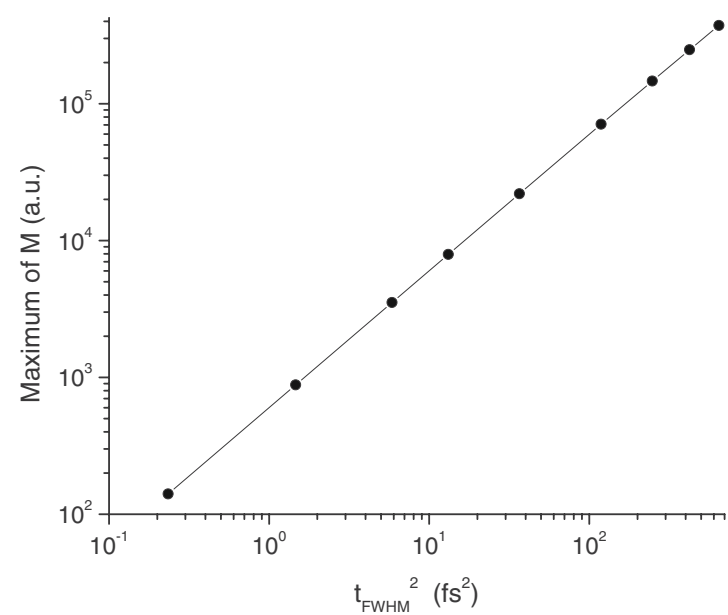

FIG. 2. The maximum of $M$ in a.u., (see text), as a function of $t_{F W H M}^{2}\left(\right.$ in $\left.\mathrm{fs}^{2}\right)$.

the main peak, which, because of the Gaussian shape of the pulse, also goes through a maximum, albeit very smoothly.

The main maximum of $P_{R}$ remains constant as a function of $t_{F W H M}$. This follows from the fact that its position is very close to the position of the resonance $\left[\varepsilon \approx 1 / q\right.$ or $E \approx E_{r}$ $+(1 / q)(\Gamma / 2)]$ and, since the photon energy is chosen to satisfy the relation $E_{i}+\omega=E_{r}$ with $t_{F W H M}$ ranging from 0.5 to 20 fs, the exponent term of Eq. (9) turns out to be $\approx 1$ for $\Gamma \tau<1$.

The above statement is equivalent to saying that the maximum of

$$
\frac{P\left(E, t \gg t_{0}+\sqrt{2} \tau\right)}{F^{2}} \equiv M
$$

is proportional to $t_{F W H M}^{2}$. Indeed, Fig. 2 exhibits this linear dependence in a quantitative way. This theoretical-

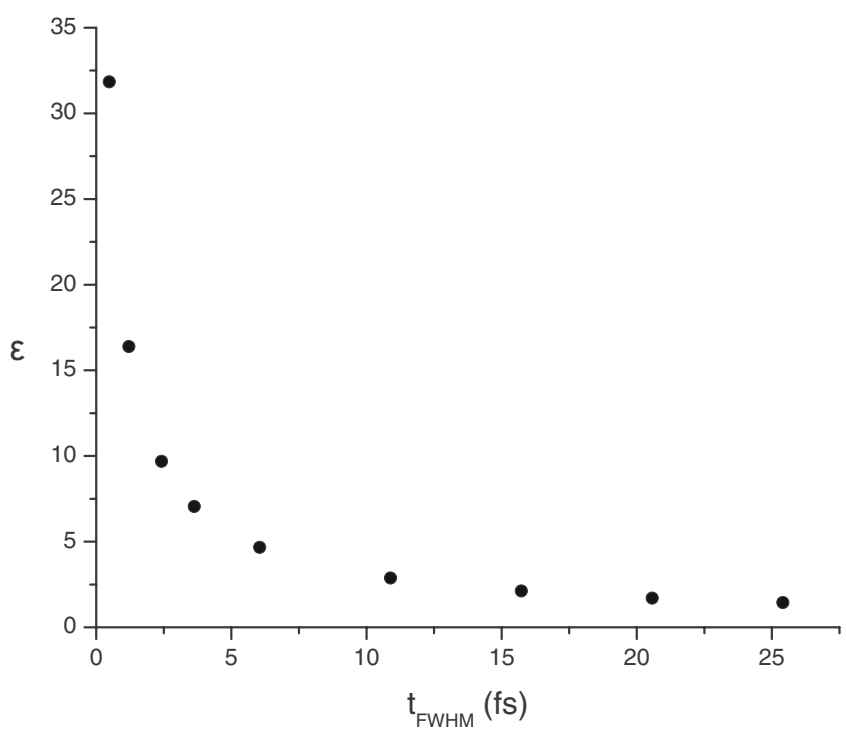

FIG. 3. The energy position of the secondary maximum of $P(E, t)$ as a function of $t_{F W H M}$ (in fs). $\varepsilon=(2 / \Gamma)\left(E-E_{r}\right)$. 
computational result can be checked and used in experimental diagnostics.

An additional new and practical finding is the fact that, in spite of its presence on a smoothly varying curve, the position of the maximum of the secondary (broad) peak can be deduced from the examination of the extrema of the expression $\frac{1}{F^{2} t_{F W H M}^{2}}\left|C_{E} G\left(E, t \gg t_{0}+\sqrt{2} \tau\right)[q+\varepsilon]\right|^{2}$. It is a matter of algebra to see that this is a function of $t_{F W H M}$ and of the resonance parameters $\Gamma, q$. In turn, this means that if one is able to measure it, then the $t_{F W H M}$ of the laser pulse can be determined.

Figure 3 shows the position of the secondary maximum as a function of $t_{F W H M}$. Its distance from the resonance position increases as the $t_{F W H M}$ decreases, while the corresponding ionization probability takes larger values. In addition, knowledge of $t_{F W H M}$ allows the direct determination of the peak intensity of the pulse (see Fig. 2), since the He $2 s 2 p{ }^{1} P^{o}$ resonance state parameters, $E_{r}, \Gamma$, and $q$, are well known $[10,12,13]$, and references there in.

In conclusion, the herein analysis and computations of the time dependence of resonance profile formation for process (1) have accomplished two things:

First, they produced information about the dynamics of the formation of resonance states excited by weak and ultrashort pulses of high photon energy.

Second, because of the accuracy of the computed results, (Figs. 1-3), and of their relevance to contemporary light sources, the proposal was made to utilize such data on resonance states toward the testing and/or improvement of the diagnostics of ultrashort pulses that are expected to be produced from the new FELs, e.g., [1-9].
[1] E. L. Saldin, E. A. Schneidmiller, and M. V. Yurkov, Phys. Rev. ST Accel. Beams 9, 050702 (2006).

[2] T. Plettner, P. P. Lu, and R. L. Byer, Phys. Rev. ST Accel. Beams 9, 111301 (2006).

[3] T. Watanabe et al., Phys. Rev. Lett. 98, 034802 (2007).

[4] A. Rudenko et al., Phys. Rev. Lett. 101, 073003 (2008).

[5] G. Lambert et al., Nat. Phys. 4, 296 (2008).

[6] B. Steffen et al., Phys. Rev. ST Accel. Beams 12, 032802 (2009).

[7] M. Röhrs, C. Gerth, H. Schlarb, B. Schmidt, and P. Schmüser, Phys. Rev. ST Accel. Beams 12, 050704 (2009); Y. Ding, Z. Huang, D. Ratner, P. Bucksbaum, and H. Merdji, ibid. 12,
060703 (2009).

[8] Y. H. Jiang et al., Phys. Rev. Lett. 102, 123002 (2009).

[9] H. Fukuzawa et al., Phys. Rev. A 79, 031201 (2009).

[10] Th. Mercouris, Y. Komninos, and C. A. Nicolaides, Phys. Rev. A 75, 013407 (2007)

[11] Th. Mercouris, Y. Komninos, and C. A. Nicolaides, Phys. Rev. A 76, 033417 (2007).

[12] U. Fano, Phys. Rev. 124, 1866 (1961); U. Fano and J. W. Cooper, ibid. 137, A1364 (1965).

[13] R. P. Madden and K. Codling, Phys. Rev. Lett. 10, 516 (1963); Astrophys. J. 141, 364 (1965). 